\title{
Penetrating Neck Injuries involving the Larynx: A Report of three cases.
}

\author{
S B. Sharma1, A 0. Amata2, \\ ${ }^{1}$ Consultant ENT Surgeon \\ ${ }^{2}$ Consultant Anaesthetist. \\ Georgetown Public Hospital Corporation, Georgetown, GUYANA, .Southerica \\ Correspondence to: Dr. Andrew 0. Amata, Email: aoamata@yahoo.com
}

Keywords: Penetrating neck injuries, laryngeal trauma, neck trauma, laryngotracheal injuries, airway trauma

Penetrating neck injuries are injuries that penetrate the platysma and are a major cause of morbidity and mortality because of the vital structures confined in the neck area. Injuries involving the larynx are especially dangerous because of the risk of airway compromise. These injuries could be intentional or accidental and the mechanisms and severity vary. Management can be challenging and sometimes controversial. We present 3 cases illustrating different mechanisms of occurrence and highlighting some of the challenging associated with management with suggestions for optimal management.

\section{Introduction}

Penetrating neck injuries (PNI) are defined as injuries that extend deep to, or penetrate the platysma. They account for approximately 5-10\% of all trauma cases presenting to the Emergency department and pose a high risk for major morbidity and mortality because of the several vital structures that traverse or reside in this relatively small area of the body. $(1,2)$ Trauma to the aerodigestive or vascular structures may be lifethreatening because of airway compromise or torrential haemorrhage. Mortality rate for PNI in civilian populations range between 3- $20 \%$ with about $50 \%$ due to exsanguinating haemorrhage from vascular injuries. (3-5) Prompt and effective management is therefore essential.The optimal management of pnis however remainscontroversial. ${ }^{(6-8)}$ We present 3 cases of PNI with associated laryngeal injuries and discuss the characteristics and challenges in their management especially in resource-constrained settings.

\section{Case Reports}

\section{Case 1.}

A 36-year-old man who was allegedly assaulted and stabbed on the front of his neck with a broken glass bottle, was initially treated at a primary healthcare centre where his trachea was intubated and then transferred to the central hospital. On arrivalat the hospital emergency department, the patient was foundto be haemodynamically stablewith no active bleeding and with no evidence of cervical spine injury. The X-ray of the neck in lateral view (Fig.1), revealed a $3 \mathrm{~mm}$ thick and $5.5 \mathrm{~cm}$ long radio-opaque shadow in the retropharyngeal space, anterior to the vertebral bodies of C3 and C4. Direct laryngoscopic examination revealed edema, hematoma, avulsed mucosa and lacerations of the epiglottis and muscles of the posterior pharyngeal wall.There was a 
bulge on the posterior pharyngeal wall produced by impaction of a large foreign body in the retropharyngeal space. Externallythe neck woundwas an oblique $5 \mathrm{~cm}$ long laceration on the anterior part of the neck starting $2 \mathrm{~cm}$ from the left side at the midpoint of the anterior border of the sternocleidomastoid muscle, and extending medially andslightly obliquely downwards across the midline of the neck to the right side about $2 \mathrm{~cm}$ above the suprasternal notch.

The laceration had penetrated the platysma to involve the medial portions of bilateral sternohyoid and thyrohyoid muscles. The injury had cut open the larynx through the thyrohyoid membrane and ligaments and extended obliquely upwards and backwards. Through the thyrohyoid space,the undamaged vocal cords could be visualized. The laryngeal injuries included lacerations of the soft tissues in the pre-epiglottic space, $1 \mathrm{~cm}$ of the tip of the epiglottis, adjoining aryepiglottic folds, mucosa and muscles of the posterior pharyngeal wall and produceda largehematoma.The injury fortunately had spared the neurovascular bundle (superior laryngeal nerve and artery) on either side and there was no active bleeding butjust oozing blood mixed with saliva. There was mild subcutaneous emphysema around thewound. An urgent surgical intervention to repair the traumatized larynx was decided.

To facilitate surgical repair of the larynx, a tracheostomy was done and the endotracheal tube removed. The larynx was repaired through the neck incision and the following structures were sutured: the lacerated tip of epiglottis and adjoining upper ends of aryepiglottic folds on both sides, the lacerated soft tissue in pre-epiglottic space, the lacerated mucosal lining in the thyrohyoid region and the thyrohyoid ligament with the perichondrium of the thyroid cartilage. Lastly the overlying platysma, subcutaneous tissues and the skin of the neck wound were closed in layers. The exploration of the retropharyngeal space for the foreign body (Fig.2) was postponed because of edema, hematoma, avulsed mucosa and lacerations of muscles of the posterior pharyngeal wall. The bulge of the posterior pharyngeal wall, produced by impaction of the large foreign body in the retropharyngeal space and the associated edema, made passage of a nasogastric tube impossible. The foreign body was removed 48 hours later after treating the patient with parenteral broad spectrum antibiotics, steroids and intravenous fluids. This was done by using a rigid laryngoscope to visualize the posterior pharyngeal wall and a horizontal incision was made over the budge caused by the foreign body at the level of the lower poles of the tonsils. The glass bottle fragment was removed in 2 pieces. (Fig.3)The posterior pharyngeal wall wound was closed with absorbable sutures. Post-operativecheck X-ray ofneck did notreveal any remnant of the foreign body.

A chest X-ray done on the $7^{\text {th }}$ post-operative day showed regression to normal of the previously widened retropharyngeal space and the tracheostomy tube was removed. The dysphagia and hoarseness improved gradually over the next two weeks. Postoperative clinic follow up over a one year period did not reveal any complications.

Case 2.

A 24-year-old young man was brought to the accident and emergency department because of agaping wound to the neck produced by a sharp metal piece while he was 
working with a welding machine.On presentation, he was conscious and haemodynamically stable but had some evidence of moderate respiratory distress. There was mild oozing of blood and air leak from the wound with associated surrounding subcutaneous emphysema. The laceration was on the left side of the anterior neck and it extended obliquely cranially along the anterior border of the left sternocleidomastoid muscle, from the suprasternal notch to $2 \mathrm{~cm}$ below the left angle of the mandible. It involved the cricothyroid membrane, the adjoining part of the cricoid and the left ala of the thyroid cartilage. The pharynx and major vessels of the neck were spared and no neurological deficit or cervical spine injury was noticed.

The patient was urgently taken to the operating room where an emergency tracheostomy was performed. Direct laryngoscopy was done to assess the laryngeal trauma which revealed edema of the glottis and lacerations at the anterior commissure from the subglottic region. The left ala of the thyroid cartilage was displaced medially producing a narrowing of the subglottic region. A laryngoplasty was done. This involved the insertion of an improvised laryngeal stent (a piece of adult size Foley's catheter, $5 \mathrm{~cm}$ long $\mathrm{x} 1 \mathrm{~cm}$ diameter)through a rigid laryngoscope between the vocal cords and extending downwards through the subglottis to rest on the top of the tracheostomy tube (Figure 4). During the exploration of the neck wound, a gap was noted $(1 \mathrm{~cm}$ size, triangular shape)between the thyroid and cricoid cartilages caused by lacerations of the cricothyroid membrane and a severed small piece from the lower margin of the left ala of the thyroid cartilage (Figure 5). This gap was bridged and stitched with a composite cartilage graft (conchal cartilage obtained from left pinna (Figure 6).The stent was fixed in the larynx by a thick nylon suture going horizontally from the left side of the larynx at subglottic level, through the skin, thyroid cartilage, the stent and then coming out from the right side of neck. On both sides of the neck it was tied on two small pieces of plastic tubes. A nasogastric tube was passed and was left in situ for 10 days. Five weeks after the surgery, the laryngeal stent was removed and 2 weeks later, the tracheostomy tube was removed. Post operatively and during post-discharge follow-up there wereno complications except for mild voice hoarseness.

\section{Case 3.}

A 39-year-old female had gunshot wounds to her neck, chin and right upper arm. When seen in the emergency room, she was spitting blood-stained saliva intermittently but was hemodynamically stable and had mild difficulty in breathing and speaking. She was receiving oxygen by facemask with satisfactory oxygenation(oxygen saturation $\sim 96$ 99\%)and was alert with moderate pain in neck and headache. She had no active bleeding, neurological deficit or any sign of cervical spine injury. There were 6penetrating gaping wounds with ragged edges, produced by the shotgun pellets; one on the left chin, two on the neck, two on the chest wall and one on the right upper arm laterally (Fig.7). The wound on the left side of the chin was $1.5 \mathrm{~cm} \times 0.75 \mathrm{~cm}$ in size, $2 \mathrm{~cm}$ below the left angle of mouth. One of the two neck wounds wason the right side of neck about $2 \mathrm{~cm}$ above the mid-point of right clavicle, $1 \mathrm{~cm}$ in size and oval in shape and the second neck wound was in the midline of the neck, $4 \mathrm{~cm}$ below chin, $1 \mathrm{~cm}$ in size, round and with lacerated edges. Of the two wounds on the chest wall, the first one was $1.5 \mathrm{~cm}$ below the lateral end of the right clavicle, $1.5 \mathrm{~cm}$ in diameter, and the other one was 
$1 \mathrm{~cm}$ lateral to the sternum and $1.5 \mathrm{~cm}$ below the medial end of right clavicle and $0.5 \times 0.25 \mathrm{~cm}$ size.The wound on right upper arm was round in shape, $1 \mathrm{~cm}$ in diameter and on the lateral aspect of the deltoid region and a hard foreign body (pellet) was felt in floor of the wound. All these wounds were surrounded by ecchymosed skin with minor bruises but no with no active bleeding. .

Though the patient was haemodynamically stable with only mild signs of respiratory distress, because of the mechanism of the injury and the multiple injury points, we decided to perform a computed tomographic angiography (CTA) to rule out any vascular or aerodigestive tract injuries. This revealed no vascular injury but 3 bullet pellets were noticed in the right side of the neck and there was a comminuted fracture of the lower edge of left mandible medially spilling multiple fragments of the bone in the floor of mouth, and elevating the floor of the mouth. There was also a comminuted fracture of the hyoid bone and midline fracture of the thyroid cartilage with its right ala displaced medially, partially blocking the laryngeal vestibule. The CTA also revealed extensive soft tissue emphysema of neck and superior mediastinum. All major blood vessels and trachea were intact.

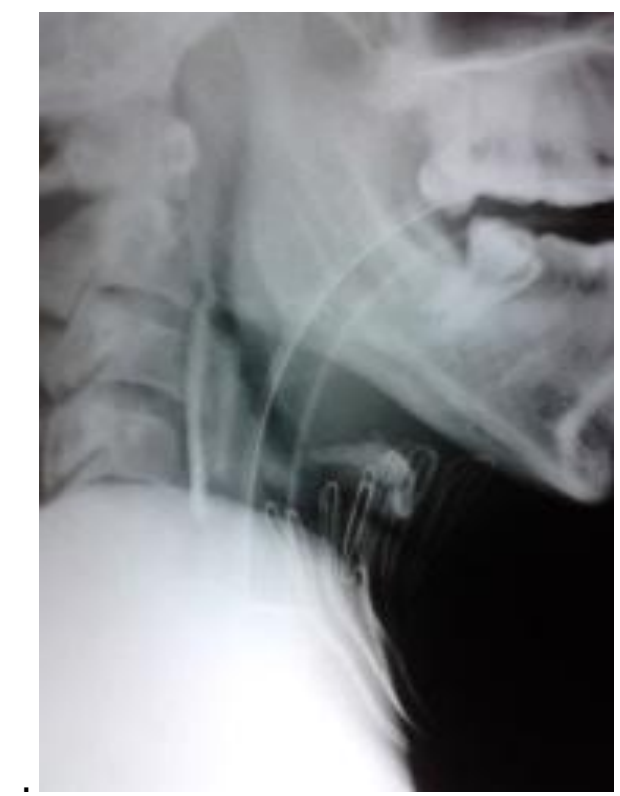

Figure 1. Preoperative X-ray Showing the Foreign Body and Endotracheal Tube

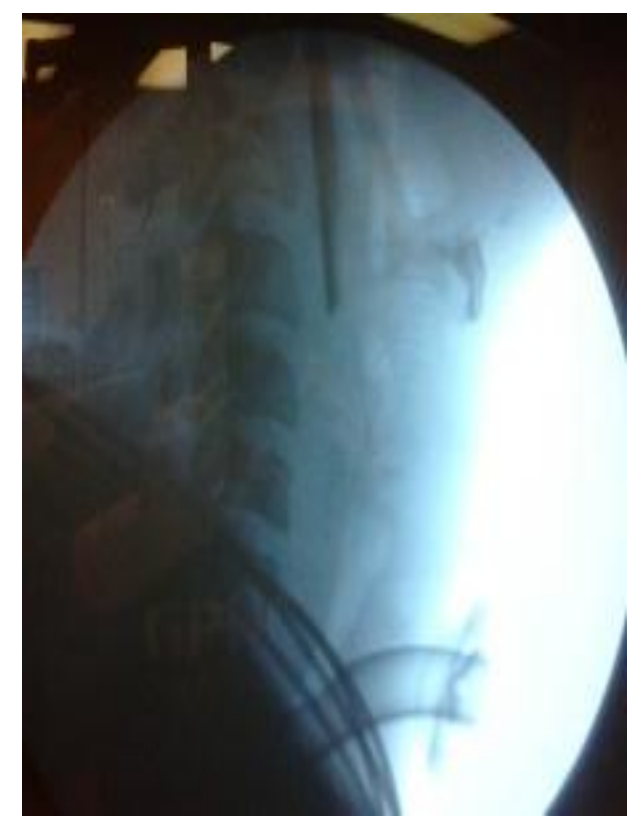

Fi Figure 2. Post Laryngeal Repair Xray-still Showing the Foreign Body 


\section{2 \\ ISSN 2073-9990 EAST CENT. AFR. J. SURG}

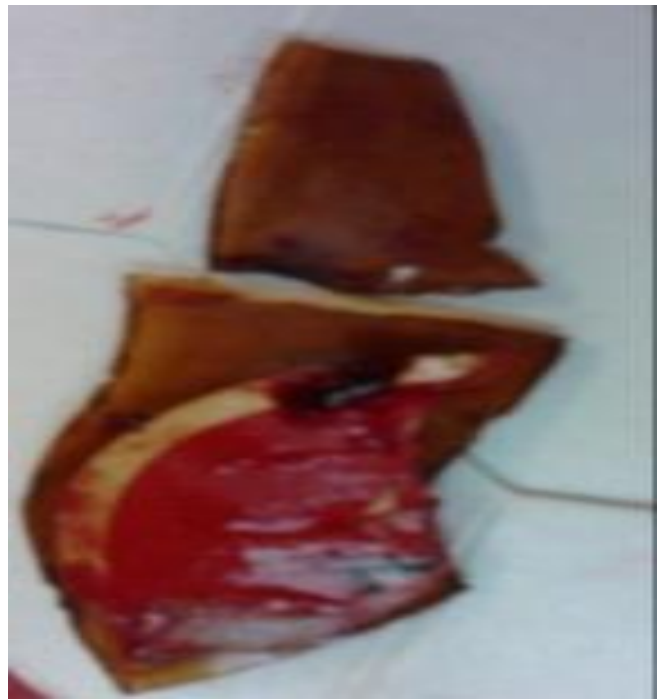

Figure 3. Broken Bottle Glass Pieces with Brand Label Still Intact

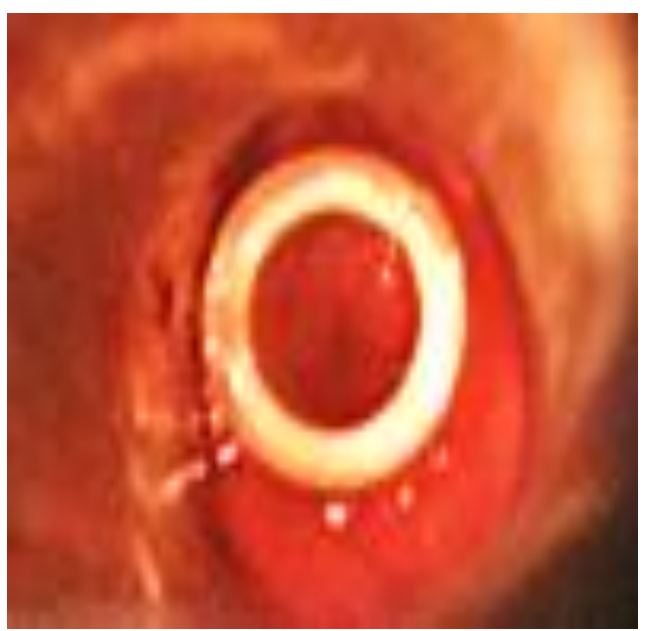

Figure 4. Improvised laryngeal stent insitu

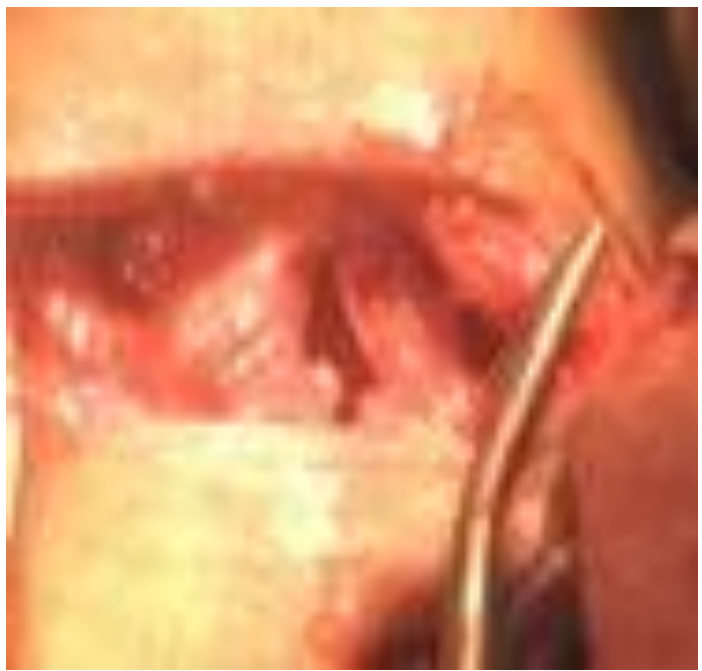

Figure 5. Laceration in the Cricothyroid Membrane

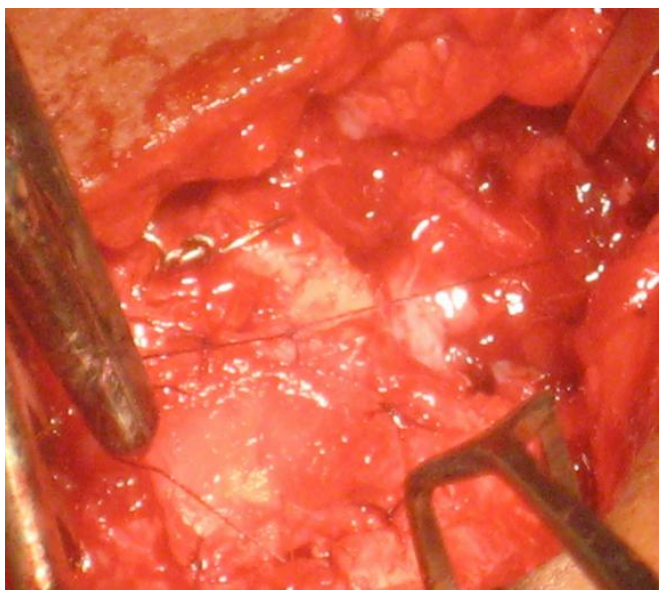

Figure 6. Composite Conchal Cartilage Graft in Position

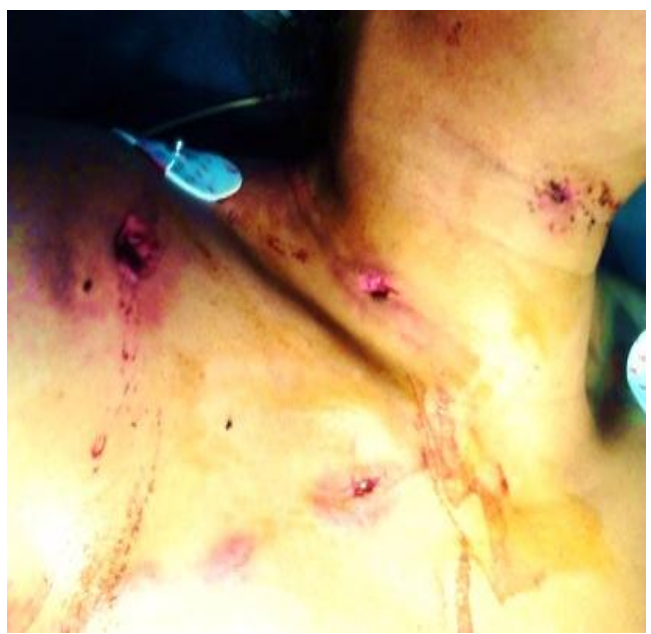

Figure 7. Penetrating wounds produced by the shotgun pellets 


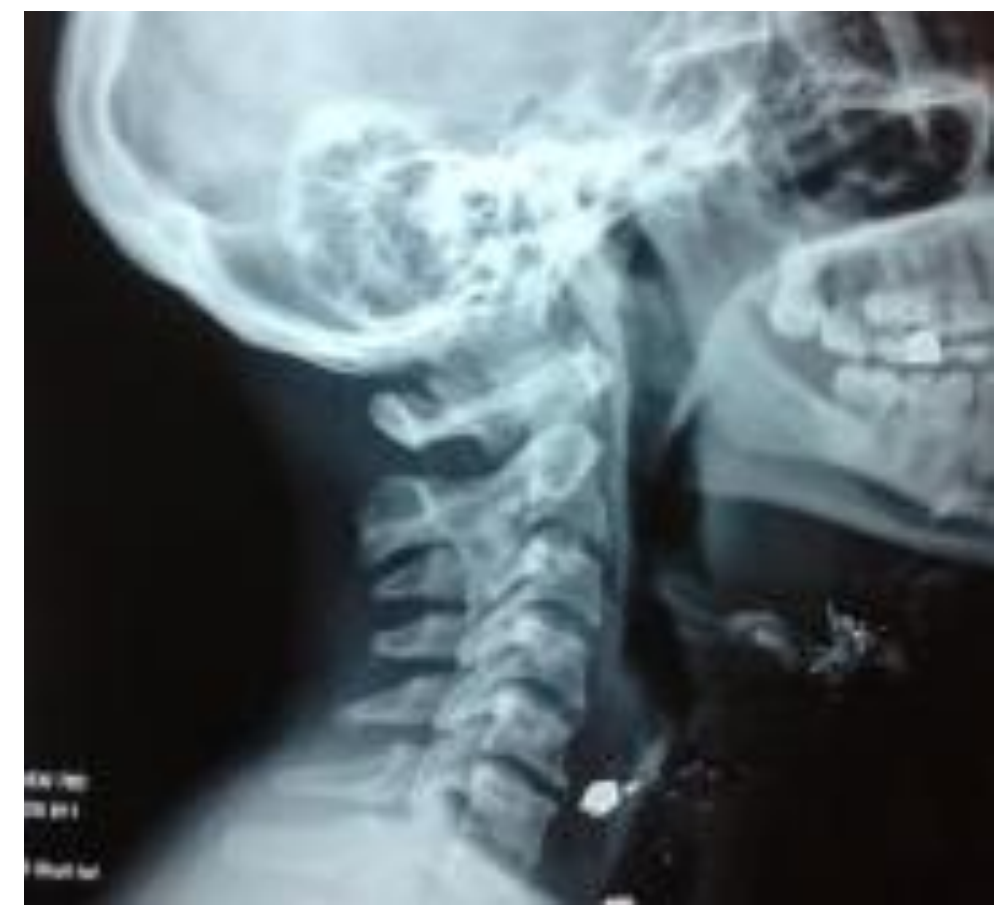

Figure 8. Check X-ray 3 Weeks after Surgery

An urgent surgical exploration under general anaesthesia with endotracheal intubation was undertaken. Exploration of the oral cavity revealed a displaced fracture of the mandible vertically between the right and left medial incisors and bleeding from the torn gingiva at the fracture site. The bleeding point was coagulated, the torn gingiva was stitched and both halves of the fractured mandible were approximated and stabilized with inter dental wiring. The wound on the chin was explored, no foreign body was palpable but a track was found going medially and downwards towards the lower border of the mandible. Through this track, the loose bone pieces were removed. Rigid esophagoscopy was done but revealed no foreign body or esophageal trauma. On exploration of the wound on the right side of the neck, a pellet was removed from the subcutaneous tissue. The midline wound of the neck was explored by extending the incision horizontally and two pellets embedded in subcutaneous tissues were removed along with a broken middle part of hyoid cartilage and few small fragments of the left mandibular fracture. To explore the fractured laryngeal cartilage, a horizontal incision was made at the level of the thyrohyoid membrane to expose the upper part of thyroid cartilage where a vertical displaced fracture of thyroid cartilage was found. The displaced lamina was realigned and then fixed in position by perichondrial stitches. The wound was closed in 2 layers with a drain on the right side of the neck and a nasogastric tube was also inserted.

On the $2^{\text {nd }}$ postoperative day enteral feeding by nasogastric tube was commenced and the endotracheal tube was removed on the third day. The patient was discharged on the fifth postoperative day after a check X-ray showed that though 2 pellets were still left in the neck, the airway was patent and well-aligned. (Fig.8) There was no dysphagia or any significant voice changes during clinic follow-up visits. 


\section{Discussion}

The neck is a relatively small area of the human body, accounting for about $3 \%$ of the total body surface area, but it encases many vital structures of most major organs or systems including the cardiovascular, respiratory, neurologic, digestive and musculoskeletal systems. The neck is commonly divided into three anatomic regions or zones as described by Roonand Christensen. ${ }^{(9)}$ Zone I is located at the base of the neck (lower neck) and extends from the clavicles to the cricoid cartilage, zone II extends cranially from the level of the cricoid cartilage to the angle of the mandible (midportion of the neck) and zoneiii is most cranial from the mandible to the base of the skull (upper neck).

Our first and second patients had injuries in zone II only and the third patient had injuries in all 3 zones and all three had laryngeal injuries. Patient 1 had injury to the epiglottic cartilage, patient 2 had injuries to the thyroid and cricoid cartilages and patient 3 had injury tothe thyroid cartilage. Most surveys and case series indicate that zone II is the most commonly affected zone in pnis ${ }^{(1,4,8)}$ and data from a large series of almost 2500 patients corroborate our findings that injury to the aerodigestive tract was often the most common, followed by the vascular system and then the nervous system in pnis.(10) However, some other studies indicate that vascular injury is the most common in pnis. ${ }^{(5)}$ These differences are probably related to the prevalent mechanism of injury in different locations. ${ }^{(1,11)}$ Approximately $50 \%$ of patients with an airway injury also have a digestive tract injury(11) but we did not observe such association in any of our cases.

Pnis like any trauma, can be classified as intentional or non-intentional. Intentional causes are mainly due to assaults or interpersonal violence and less commonly to suicidal attempts or self-mutilation. Non-intentional causes are mainly accidents such as vehicular, domestic and occupational accidents. Injuries in patients 1 and 3 were intentional (assault) while patient 2 was accidental (work-related). Objects causing pnis include stabbing instruments such as knives, forks and broken glass or bottles, cutting instruments such as razor blades, and sharp edges, and missiles or projectiles such as guns, arrows, and spears. The pattern of pnis vary in different regions or environment. ${ }^{8}$, 11) In urban centres and countries with liberal gun laws such as the USA, gunshot wounds (gsws) may be more frequent compared to more rural areas or countries with strict gun laws. In a recent review of large case series from the USA, Canada and South Africa,(11) stab wounds (sws) was the most common type of PNI (70\%) with GSW a distant second at $22 \%$ and others types, $8 \%$. Sws included injuries with a knife, razor blade or bottle while gsws were due to either handguns or shotguns.

The severity of PNI often correlates with the mechanism of injury with high velocity injuries such as those caused by guns or speeding automobiles being more likely to produce more extensive damage. $(8,10)$ Patient 3 with GSW had injuries to all 3 zones of the neck and in addition had other associated injuries including wound to the upper limb, chin and jaw. About $30 \%$ to $80 \%$ of patients with pnis have other injuries outside of the neck region. $(1,2,4)$ 
in all trauma cases, management should follow the Advanced Trauma Life Support (ATLS) approach developed by the American College of Surgeons ${ }^{(12)}$ with emphasis on ensuring a patent airway, adequate oxygenation and ventilation, and optimization of the blood circulation. Priority must be given to securing the airway especially in patients with actual or potential airway injuries like ours. A definitive airway usually by endotracheal intubation is preferably and commonly used $(5,711-14)$ as was done in patients 1 and 3.In stable cases with no obstruction to the airway, oxygenation can be improved by appropriately positioning the patient and administering oxygen by facemask. However when significant laryngotracheal injury is suspected, endotracheal intubation should be done cautiously to prevent further damage.(11,13,14) If available, direct visualization of the tracheobronchial tree and endotracheal intubation via a fibreoptic bronchoscope may be preferred.(14)If endotracheal intubation is not feasible or suitable a surgical airway may be necessary. A cricothyrotomy is safe and fast but if the injury has disrupted the larynx then a tracheostomy should be done. A tracheostomy, apart from assuring a patent airway, facilitates laryngo-tracheal repairs-(13, 14) Our $1^{\text {st }}$ and $2^{\text {nd }}$ patients had tracheostomy. In the first case, an initial endotracheal intubation was done and this was later exchanged for a tracheostomy, prior to the laryngeal repair. The tracheostomy was required for 7 days in case1before decannulation but in case 2 it had toremain for 5 weeks as a laryngeal stent was put in the repaired larynx. Postoperatively there was no permanent change in voice, difficulty in breathing or swallowing problems in any of our patients. Injury to the cervical vertebrae has been noted to be rare in pnis ${ }^{(13)}$ and unless there is evidence of or a high index of suspicion of cervical spine injury, it is recommended that cervical collars be not routinely used as these may obscure examination of the neck. ${ }^{(11,13)}$

Once primary assessment, necessary resuscitation and stabilization has been done, the next important decision is the modality of definitive management. Controversy still persists about the optimal management of pnis. Some surgeons insist on mandatory surgical exploration of all pnis while others opt for a selective surgical approach. In the last 2 decades there has been a gradual shift in favour of the selective surgical approach. ${ }^{(1,2,11)}$ Rationale for this shift include the very high rate of negative surgical explorations (up to $90 \%$ in some studies) (1-3) and associated complications, the relatively low mortality rate in PNI and the increasing availability of modern diagnostic modalities $(6,8)$ Many systematic reviews and audits have shown that there is no clinically significant difference in outcomes between patients who had mandatory exploration and those who had selective management. $(1-5,8,11,14,15)$

Careful physical examination has been shown to be safe and reliable in predicting the need for surgical exploration or further diagnostic investigations. ${ }^{(16)}$ Most pnis that were significant and required surgical exploration were symptomatic on presentation. (1-4, 11)Clinical findings are usually classified as "hard" or "soft" $(8,11,15)$ Hard signs are indicative of major vascular or aerodigestive tract injury and include active bleeding, haemodynamic instability, expanding or pulsatile haematoma, bruit/thrill, pulse deficit, central neurologic deficit/evolving stroke, refractory shock, massive subcutaneous emphysema and significant airway compromise. Soft signs include venous oozing, nonexpanding or non-pulsatile haematoma, minor haemoptysis, dysphonia, dysphagia, air bubbling through wound, and mild emphysema. Patients with hard signs require 
immediate surgical intervention while patients with soft signs may require further investigations to validate the need or not for surgical exploration. Asymptomatic haemodynamically stable patients often just require careful observation. Patients with injuries to the respiratory tract often present with signs and symptoms of hoarseness, haemoptysis, odynophagia, stridor and subcutaneous emphysema. (6,7) All 3 of our patients had mild to moderate respiratory distress, subcutaneous emphysema, bloodtinged saliva or spittle and air leakfrom the neck wound. Fortunately all 3 patients were haemodynamically stable and had no active bleeding. Thus their surgical interventions to correct the laryngotracheal injuries, while urgent, did not have to be done immediately, allowing time for diagnostic tests to further assess the injuries.

Endoscopy (direct laryngoscopy and esophagoscopy) was performed in all 3 cases because of concerns of aerodigestive tract trauma as evidenced by salivary contamination of the wound site, airleak from the wound, presence of foreign body (glass piece and pellets)close to the oesophagus orin the retropharynx and the associated symptoms of dysphagia and neck pain. None of our patients had any esophageal injuries.

Modern imaging techniques have improved the diagnosis and management of pnis and significantly reduced negative surgical neck explorations. ${ }^{17) C T A}$ especially the recent multi-detector computed tomographic angiography (MDCTA) is fast, non-invasive, reliable and accurate in the assessment of both vascular and aerodigestive tract injuries and is now considered the preferred imaging modality in pnis. (17) We used it in our $3^{\text {rd }}$ case because of the high suspicion of injuries to vital structures.

Our findings and management support and corroborate the algorithms and guidelines recommended by Nason and colleagues, (1) Demetriades and colleagues ${ }^{(2)}$ and the Western Trauma Association.(15) Patients will obvious significant injuries or with hard signs suggestive of major vascular or aerodigestive tract injuries should proceed immediately to surgical exploration. Stable and asymptomatic patients can be managed by serial observations while stable patients with evidence of soft signs should have investigations such as CTA, laryngoscopy, esophagoscopy and surgery if necessary. In resource constrained settings where facilities and expertise may be lacking,more liberal utilization of surgical exploration is reasonable. (3)

\section{Conclusion}

Pnis comprise a small proportion of trauma emergencies and can be challenging to manage. Mandatory surgical exploration is no longer favoured. Selective surgical management is now preferred. Modern reliable diagnostic modalities have improved diagnosis and reduced rates of negative surgical explorations. Ultimately, the decision to explore surgically would be influenced by the skills and experience of the surgeon and the resources available at each particular institution. In centres with very limited resources, it may be safer to err on the side of more liberal surgical explorations. 


\section{Informed Consent}

This report received institutional ethics approval and all the three patients gave informed consent and provided written permission for publication of the case reports.

\section{References}

1. Nason RW, Assuras GN, Gray PR, Lipschitz J, Burns CM. Penetrating neck injuries: Analysis of experience from a Canadian trauma centre. Can J Surg. 2001; 44 (2):122-6.

2. Demetriades D, Asensio JA, Velmahos G, Thal E. Complex problems in penetrating neck trauma. Surg Clin North Am. 1996; 6 (4):661-83

3. Gilyoma JM, Hauli KA, Chalya PL. Cut throat injuries at a university teaching hospital in northwestern Tanzania: a review of 98 cases. BMC Emergency Medicine 2014 14:17.DOI:10.1186/1471-227X-14-1

4. Al-Thani H, El-Menyar A, Mathew S, et al. Patterns and outcomes of traumatic neck injuries: A population-based observational study. Journal of Emergencies, Trauma, and Shock. 2015; 8(3):154-158. Doi:10.4103/0974-2700.160723.

5. Mahmoodie M, Sanei B, Moazeni-Bistgani M, Namgar M. Penetrating neck trauma: Review of 192 cases. Arch Trauma Res. 2012;1:14-8

6. Asensio JA, Valenziano CP, Falcone RE, Grosh JD., Management of penetrating neck injuries. The controversy surrounding zone II injuries, thesurgical Clinics of North America. 1991 Apr;71(2):267-96.

7. Akhtar S, Awan S. Laryngotracheal trauma:its management and sequelae. J Pak Med Assoc 2008;58:241-3.

8. Harris $\mathrm{R}$, Olding $\mathrm{C}$, Lacey $\mathrm{C}$ et al. Changingincidence and management of penetrating neck injuries in the South East London trauma centre. Ann R Coll Surg Engl 2012:94;240-4

9. Roon AJ, Christensen N. Evaluation and treatment of penetrating cervical injuries. J Trauma 1979;19(6):391-7.

10. Mcconnel DB, Trunkey DD. Management of penetrating trauma of the neck. Adv Surg 1994; 27:97-127.

11. Burgess CA, Dale OT Almeyda R, Corbridge RJ, An evidence based review of the assessment and management of penetrating neck trauma.Clinical Otolaryngology. 2012 Feb;37(1):44-52. Doi: 10.1111/j.1749-4486.2011.02422.x.

12. American college of Surgeons, Advanced Trauma Life Support Program for Physicians. $9^{\text {th }}$ ed. Chicago, IL: 2012. Www.facs.org/quality\%20programs/trauma/atls

13. Brywczynski لU, Barrett TW, Lyon JA, Cotton BA, Management of penetrating neck injury in the emergency department: a structured literature review. Emergency 2008;25:711-5. Doi: 10.1136/emj.2008.058792.

14. Shearer VE, Giesecke AH. Airway management for patients with penetrating neck trauma: a retrospective study. Anesth Analg. 1993 Dec; 77(6):1135-8.

15. Sperry JL, Moore EE, Coimbra R et al. Western Trauma Association Critical Decisions in trauma:penetrating neck trauma. J Trauma Acute Care Surg 2013; 75 (6): 936-40. Doi: 10.1097/TA.0b013e31829e20e3

16. Ahmed A. Selective observational management of penetrating neck injury in northern Nigeria. S Afr J Surg. 2009; 47(3):80, 82-5

17. Offiah C, Hall E. Imaging assessment of penetrating injury of the neck and face.Insights Imaging. 2012;3(5):419-31. Doi: 10.1007/s13244-012-0191-y. 